Classification

Physics Abstracts

$36.20 \mathrm{E}-05.40-82.70 \mathrm{G}$

\title{
Dilution and polydispersity in branched polymers
}

\author{
M. Daoud (*), F. Family (**) and G. Jannink $\left(^{*}\right)$ \\ (*) Lab. Léon Brillouin, C.E.N. Saclay, 91191 Gif sur Yvette Cedex, France \\ (**) Department of Physics, Emory University, Atlanta, GA 30322, U.S.A.
}

(Reçu le 13 septembre 1983, révisé le 25 novembre, accepté le 9 janvier 1984)

\begin{abstract}
Résumé. - Des propriétés expérimentalement intéressantes d'un sol dilué par un bon solvant sont étudiées. La dilution laisse la distribution en masse inchangée mais conduit à un gonflement de chaque polymère branché, et donc à un changement de propriétés mesurées telles que le rayon moyen $\left\langle R^{2}\right\rangle_{z}$, le coefficient de diffusion $\langle D\rangle_{z}$ et la viscosité intrinsèque. Nous comparons les résultats pour des échantillons dilués à ceux de la polycondensation (percolation) et trouvons une différence aisément mesurable. Le gonflement décrit par une théorie de Flory.
\end{abstract}

\begin{abstract}
Experimentally relevant properties of a sol diluted in a good solvent are studied. Dilution leaves the molecular weight distribution unchanged, but leads to a swelling of every branched macromolecule, and thus to a change in the properties that are measured such as the mean square radius $\left\langle R^{2}\right\rangle_{z}$, the diffusion coefficient $\langle D\rangle_{z}$ and the intrinsic viscosity $\langle[\eta]\rangle$. We compare the results for diluted samples with those for polycondensation (percolation) and find an experimentally distinguishable difference between them. Swelling is described by a Flory theory.
\end{abstract}

\section{Introduction.}

Recently much attention has been focused on theoretical [1-7] and experimental $[7,8]$ investigations of randomly branched polymers. Theoretically, two cases have been considered : diluted monodispersed solutions, and very broad molecular weight distributions found in the polycondensation of multifunctional monomers. This last case was studied in the reaction bath and has been related to the sol-gel transition [1,2]. As pointed out by de Gennes [1] and by Isaacson and Lubensky [2], the main difference between these two cases lies in the screening of the excluded volume interaction in the polycondensed system. It is however possible to consider a 3rd case in which the excluded volume interaction is not screened in the polycondensed system. This is the situation where the chemical reaction is allowed to proceed to a certain stage below the gel point. At this point the reaction is stopped by freezing or deactivation of the reacting groups and the sample is diluted by a known quantity of solvent. Clearly, this process does not correspond to either a monodispersed solution or a polycondensed system. It corresponds exactly to the experimental situation.

In this letter we consider the experimentally interesting case of a diluted polycondensed branched polymer solution. We determine the average radius of gyration, the diffusion coefficient, and the intrinsic viscosity which are measured by means of neutron scattering, light scattering and 
viscosity measurements. We find that the results are much different from those for undiluted polycondensed solutions (with a percolation distribution) and should be measurable experimentally. The whole approach is at the level of a Flory theory $[1,2,6,7,10]$. This is known $[1,2,6,7,10]$ to give a fairly good description of reality.

\section{Earlier results.}

1. 1 Single POLYMER. - Flory theory $[9,10]$ gives the following result for the radius of gyration $R$ of a randomly branched polymer of $N$ monomers of length $I$ (« animal »).

$$
R \sim l N^{v}
$$

with $[2,6]$

$$
v=\frac{5}{2(d+2)}
$$

in a good solvent, and [6]

$$
v_{\theta}=\frac{7}{4(d+1)}
$$

in a theta solvent. Both these results are valid for a single branched polymer so that there is no dispersity in the problem thus far.

1.2 Sol. - Consider a system of multifunctional units that are allowed to react until some point below the gel point. Let $p$ be the probability that a link is made between two neighbouring monomers, $p_{\mathrm{c}}$ the gelation threshold and $\varepsilon=p-p_{\mathrm{c}}$. Suppose the reaction is quenched at a given $\varepsilon$. Then the distribution of sizes is [11]

$$
P(N)=N^{-\tau} f\left(\varepsilon N^{\sigma}\right)
$$

where $P(N)$ is the number of $N$-mers, $\tau$ and $\sigma$ exponents which are related to percolation exponents [11]

$$
\sigma=\frac{1}{\beta+\gamma}, \quad \tau=2+\frac{\beta}{\beta+\gamma} .
$$

In three dimension, $\beta \cong 0.4$ and $\gamma \cong 1.8$. Note that in the Flory-Stockmayer theory [9] $\beta=\gamma=1$. As far as the radius of gyration is concerned, the only difference between the present case and the single polymer problem lies in the screening of interactions in a Flory approach $[1,2]$.

\section{Diluting the sol.}

Let us consider the system described in section 1.2 and stop the reaction below $p_{\mathrm{c}}$ at some point $\varepsilon=p-p_{\mathrm{c}}$. If we dilute the sol in a good solvent, two effects occur simultaneously.

a) The distribution function remains unchanged, and thus the weight average molecular weight $N_{\mathrm{w}}$ is a function only of the point where the reaction was quenched :

$$
N_{\mathrm{w}} \sim \frac{\int N^{2} P(N) \mathrm{d} N \sim \varepsilon^{-\gamma}}{\int N P(N) \mathrm{d} N}
$$


b) Screening is no longer present, because the system is now dilute. Every individual molecule is swollen and within a Flory theory it is argued that the polymer reaches the " animal " behaviour (Eq. (1)) :

$$
R(N) \sim N^{v} l \text { with } v=\frac{5}{2(d+2)}
$$

The quantities that may be determined by neutron and light scattering experiments are the radius of gyration and the diffusion constant. Then what is measured is a $z$-average [9]

$$
\langle A\rangle_{z}=\frac{\int A(N) N^{2} P(N) \mathrm{d} N}{\int N^{2} P(N) \mathrm{d} N}
$$

Another set of experiments concerns the intrinsic viscosity. In this case what is measured is a weight average [7]

$$
\langle[\eta]\rangle=\frac{\int[\eta(N)] N P(N) \mathrm{d} N}{\int N P(N) \mathrm{d} N} .
$$

In such a dilute solution it seems reasonable to assume that hydrodynamic interactions are present and a Zimm type behaviour is valid for the diffusion constant and the intrinsic viscosity [13] of a macromolecule :

$$
\begin{gathered}
D(N) \sim R^{-1}(N) \sim N^{-v} \\
{[\eta(N)] \sim R^{d} / N \sim N^{d_{v}-1}}
\end{gathered}
$$

Combining [2-9] we find :

$$
\begin{aligned}
& \left\langle R^{2}\right\rangle_{z} \sim N_{\mathrm{w}}^{2 v(1+\beta / \gamma)} \\
& \langle D\rangle_{z} \sim N_{\mathrm{w}}^{-v(1+\beta / \gamma)} \\
& \langle[\eta]\rangle_{\mathrm{w}}^{3 v(1+\beta / \gamma)-1-\frac{2 \beta}{\gamma}} .
\end{aligned}
$$

The values of the exponents for the three dimensional case are given in table I. In the second row of the table we give the values for the polycondensed system, i.e. without taking swelling due to dilution into account. This corresponds to the percolation case which is usually used to compare with experiments [7]. Note that dissolving in a theta solvent leads to a similar effect, except $v$ is replaced by $v_{\theta}$ in equations $(10-12)$.

If the same experiment is to be performed above the gelation threshold, then one must assume that it is possible to separate the sol from the gel completely. This is a strong but reasonable, hypothesis [12]. Then it is possible to relate the quantities calculated to the gel fraction $G$,

$$
G \sim|\varepsilon|^{\beta}
$$

where $\beta$ is a percolation exponent used before. Combining (4) with (13) gives

$$
G \sim N_{\mathbf{w}}^{-\beta / \gamma}
$$


Table I. - Values of the exponents $x$ for the weight average molecular weight $N_{\mathrm{w}}$ dependence of the various quantities indicated, $\langle A\rangle \sim N_{\mathrm{w}}^{x}$, for three dimensional systems. The first line assumes swelling after dilution. The second line gives the results for polycondensation without dilution (i.e. percolation). For these first two lines percolation distribution is assumed. The last two lines give the same results supposing that the mean field Flory-Stockmayer theory is valid.

\begin{tabular}{|l|c|c|c|}
\cline { 2 - 4 } \multicolumn{1}{c|}{} & $\left\langle R^{2}\right\rangle_{z}$ & $\langle D\rangle_{z}$ & $\langle[\eta]\rangle$ \\
\hline Diluted system & 1.26 & -0.63 & 0.37 \\
\hline Percolation & 1.00 & -0.50 & 0 \\
\hline Diluted classical & 2.00 & -1 & 0 \\
\hline F.S. & 1.00 & -0.50 & 0 \\
\hline
\end{tabular}

which may also be used to check experimentally the " criticality " of the experiments. Combining now (14) with (10)-(12) gives

$$
\begin{aligned}
\left\langle R^{2}\right\rangle_{z} & \sim G^{-2 v(1+\gamma / \beta)} \\
\langle D\rangle_{z} & \sim G^{v(1+\gamma / \beta)} \\
\langle[\eta]\rangle^{-3 v(1+\gamma / \beta)+2+\gamma / \beta} & \sim G^{-}
\end{aligned}
$$

The values of the exponents are listed in table II for the diluted case and the undiluted polycondensed case. Again, there is a clear difference between the two cases which should be observable experimentally.

\section{Conclusion.}

Upon dilution in a good solvent the branched molecules constituting a sol should swell. The molecular weight distribution is however unchanged. This leads to a mixing of the properties of a single chain (swelling) with the distribution of sizes in percolation. Swelling has been described here within a Flory approach.

Table II. - Values of the exponent $y$ for the gel fraction $G$ dependence of the various quantities indicated, $\langle A\rangle \sim G^{y}$, for three dimensional systems. The different lines are the same as in table 1. These may be measured only above the threshold. We suppose that it is possible to separate completely the gel from the sol.

\begin{tabular}{|l|c|c|c|}
\cline { 2 - 4 } \multicolumn{1}{c|}{} & $\left\langle R^{2}\right\rangle_{2}$ & $\langle D\rangle_{2}$ & $\langle[\eta]\rangle$ \\
\hline Diluted system & -4.87 & 2.43 & -1.43 \\
\hline Percolation & -3.87 & 1.94 & 0 \\
\hline Diluted classical & -2.00 & 1.00 & 0 \\
\hline F.S. & -1.00 & 0.50 & 0 \\
\hline
\end{tabular}


A similar effect is expected if we suppose that the weight distribution is given by the classical Flory-Stockmayer (F.S.) theory rather than by percolation. The results are also given in tables I and II for this distribution and differ from those quoted above only by the values of $\beta$ and $\gamma$ which are both equal to unity in the F.S. theory, and they are also markedly different from those of the undiluted sol, i.e. F.S., as also shown in the tables. The result is that the power laws in the two cases (diluted and undiluted sol) are different and this difference is experimentally measurable. Recent experiments by Schmidt and Burchard [8] and Bantle et al. [14] agree with the swelling law corresponding to the distribution of sizes in the percolation model. On the other hand, we expect the classical F.S. exponents to describe the distribution of molecular weight in the vulcanization case.

\section{Acknowledgments.}

The authors are much indebted to L. de Sèze for stimulating discussions and S. Candau for sending his preprint. Research of F.F. was supported by grants from the Research Corporation, Emory University Research Fund and N.S.F. grant No D.M.R. 82-08051.

\section{References}

[1] De Gennes, P. G., C.R. Hebd. Séan. Acad. Sci. 291 (1980) 17.

[2] IsaAcson, J., Lubensky, T. C., J. Physique Lett. 41 (1980) L-469.

[3] Redner, S., J. Phys. A 12 (1979) L239.

[4] Derrida, B., HerrmanN, H. J., Saclay preprint.

[5] Family, F., J. Phys. A 13 (1980) L 325.

[6] Daoud, M., Joanny, J. F., J. Physique 42 (1981) 1359.

[7] Stauffer, D., Coniglio, A., Adam, M., Adv. Poly. Sci. 44 (1982) 103.

[8] SCHMidT, M., BuRCHARD, W., Macromolecules 14 (1981) 370. Munch, J. P., ANKrim, M., Hild, G., OKasha, R. and Candau, S., to be published.

[9] Flory, P. J., Principles of Polymer Chemistry (Cornell Univ. Press) 1953.

[10] De Gennes, P. G., Scaling Concepts in Polymer Physics (Cornell Univ. Press) 1979.

[11] Stauffer, D., J. Chem. Soc. Faraday Trans II 72 (1976) 1354.

[12] Gordon, M., Kajiwars, K., Peniche-Covas, C. A. L., Ross-Murphy, S. B., Makromol. Chem. 176 (1975) 2413.

[13] SieVers, D., J. Physique Lett. 41 (1980) L-535.

[14] Bantle, S., Hasslin, H. W., Ter Meer, H. U., Schmidt, M. and Burchard, W., Polymer. 23 (1982) 1889. 